concurrent immunomodulatory therapy and there are no clear predictors of efficacy. Analysis of transcriptome from peripheral blood CD4 and CD8 T-cells has been shown to predict disease outcome in inflammatory bowel diseases (IBD) but this strategy has not been tested to predict response to biological therapy. We investigated the utility of baseline CD4 and CD8 transcriptome in predicting response to anti-TNF agents in UC.

Methods Patients who were commenced on any anti-TNF therapy for ambulant UC were included in this single centre cohort study. Clinical response and remission was defined using full or partial Mayo score at week 14. RNA was extracted from peripheral blood CD-4 and CD-8 populations and subjected to transcriptome analysis using human Clariom D analysis. Transcriptome Analysis Console (TAC) 4.0 from ThermoFisher Scientific was used to analyse Expression Array feature intensity (CEL) files. The analysis was carried out with the Clariom_D_Human NetAffx Library. Statistical analysis to detect differential expressed genes was carried out with default settings of TAC, except that the use of FDR p-values was set from false to true.

Results Ten patients with UC with a median age of 35 (range 19-69) and median Mayo score of 8 (range 2-12) were included. Three (30\%) had pancolitis and $6(60 \%)$ of patients were on concomitant immunomodulators. At week 14, six $(60 \%)$ and $4(40 \%)$ patients achieved clinical response and remission respectively. Of the 135,750 genes tested, differential expression was noted in over 900 genes between responders and non-responders at a $\mathrm{P}$ value of $<0.05$. However, there was only one differentially expressed gene in the CD4 cell population in patients who achieved clinical remission with an FDR P-val < 0.05. There was a 25.87 fold higher expression of the major histocompatibility complex, class I, U (pseudogene) in patients who failed to achieve remission. Similarly, HLA-U pseudogene expression at baseline was higher in patients with lack of clinical response (12 fold vs 7 fold, $\mathrm{P}<0.01$ ). Receiver operating characterestic (ROC) analysis for HLA-U pseudogene predicted clinical remission with $>95 \%$ sensitivity and specificity $(\mathrm{P}<0.0001$, figure 1$)$.

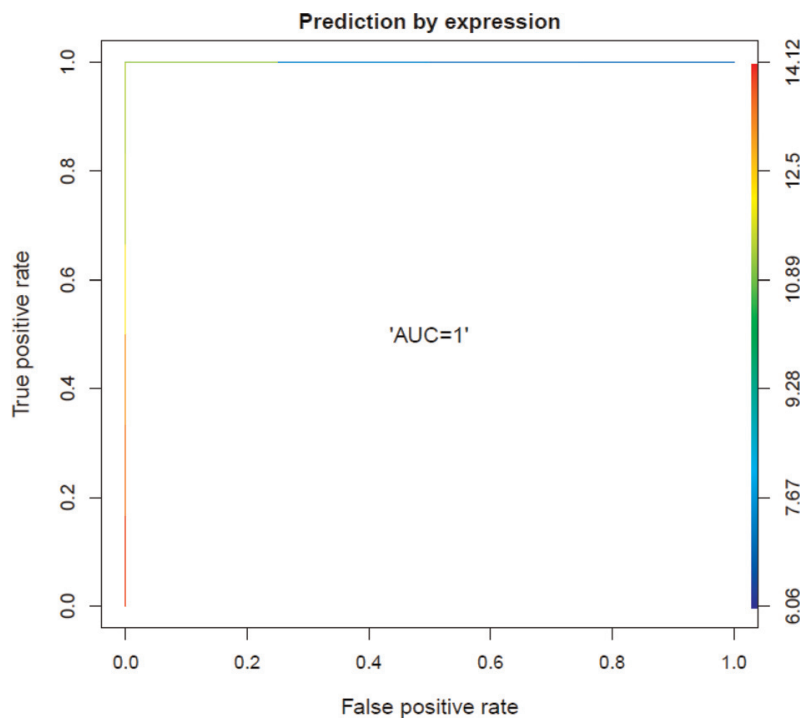

Abstract OWE-37 Figure 1 ROC curve of HLA-U psudogene to predict clinical remission to anti-TNF agents at week 14
Conclusions CD4 transcriptome analysis at baseline identified differentially expressed genes in patients with lack of clinical remission. This has potential utility as a novel non-invasive biomarker of resposne to anti-TNF therapy in UC. Our findings require further validation in a larger cohort.

\section{OWE-38 INFLIXIMAB INDUCTION REGIMES IN STEROID REFRACTORY ACUTE SEVERE COLITIS: MULTI-CENTRE STUDY WITH PROPENSITY SCORE ANALYSIS}

Shaji Sebastian*, Sally Myers, Sreedhar Subramanian, ELEVATE UC Study Group. Hull University Teaching Hospitals NHS Trust, Hull, UK

\subsection{6/gutjnl-2019-BSGAbstracts.128}

Background While infliximab is used as rescue therapy for steroid refractory acute severe colitis (ASUC), between 30 and $40 \%$ of patients do not respond and undergo colectomy. Accelerated induction regimes of infliximab have been proposed to improve response rates. We aimed to evaluate colectomy rates in steroid refractory ASUC patients receiving standard induction (SI) vs. accelerated induction (AI) of infliximab.

Methods Data collected on hospitalised patients receiving rescue therapy for steroid refractory ASUC. The choice of rescue therapy was at the discretion of the treating clinician. Accelerated induction (AI) was defined as receiving second dose of infliximab within 8 days of first rescue therapy or receiving front loading dose of $10 \mathrm{mg} / \mathrm{kg}$. Our primary outcome was the short-term (in-patient, 30 days and 90 days) colectomy rate. Secondary outcomes were 12-month colectomy rates, length of hospital stay (LOS), and complication rates. We used a propensity score analysis with optimal calliper matching using a priori defined high-risk covariates at the start of rescue therapy (albumin, CRP, CRP-albumin ratio, haemoglobin nadir and pancolitis) to reduce potential provider selection bias.

Results A total of 131 patients receiving infliximab rescue therapy were included, of whom 102 patients received SI and 29 received AI. There was no difference in age, duration of diagnosis, age at rescue therapy, Montreal class or use of steroids, 5ASAs or thiopurines prior to index admission. In the unmatched overall cohort, there was no difference in colectomy during index admission ( $13 \%$ vs. $20 \%, \mathrm{p}=0.26), 30$ day colectomy $(18 \%$ vs. $20 \%, p=0.45)$, 90 -day colectomy $(20 \%$ vs. $24 \%, p=0.38)$ or 6 month colectomy $(25 \%$ vs. $27 \%, p=0.49)$. The LOS was shorter in the SI group $(14.87 \pm 8.1$ days vs. $19.31 \pm 5.8$ days, $p=0.007)$. In patients who underwent colectomy, there were no differences in complications or serious infection rates. In the propensity score-matched cohort of 52 patients, there was no difference in overall colectomy rates between SI and AI groups $(57 \%$ vs. $31 \%, \mathrm{p}=0.09)$, but the index admission colectomy $(53 \%$ vs. $23 \%, \mathrm{p}=0.045)$ and 30 -day colectomy $(57 \%$ vs. $27 \%, \mathrm{p}=$ 0.048 ) rates were higher in those receiving SI. There was no significant difference in LOS between SI and AI groups (23.6 \pm 4.3 vs. $18.2 \pm 7.1$ days, $p=0.09$ ) or in overall complication and infection rates but there was a mortality in AI group.

Conclusion In this retrospective cohort study, there was no difference in overall colectomy rates in ASUC patients 
receiving different induction dosing regimens of infliximab. However, using propensity score matching, the short-term colectomy rates appear to be better in those receiving accelerated induction regime. A prospective study to confirm findings is planned.

\section{Abstracts of Distinction}

\section{ATH-07 PRE-MORBID DEPRESSION AMONGST INDIVIDUALS SUBSEQUENTLY DEVELOPING INFLAMMATORY BOWEL DISEASE: A POTENTIAL CO-FACTOR MEDIATING DISEASE ONSET?}

${ }^{1}$ Jonathan Blackwell ${ }^{*}{ }^{2}$ Sonia Saxena, ${ }^{1}$ Christopher Alexakis, ${ }^{2}$ Elizabeth Cecil, ${ }^{2}$ Alex Bottle, ${ }^{3}$ Irene Petersen, ${ }^{4}$ Matthew Hotopf, ${ }^{1}$ Richard Pollok. ' St George's NHS Foundation Trust, London, UK; ${ }^{2}$ Imperial College London, London, UK; ${ }^{3}$ University College London, London, UK; ${ }^{4}$ King's College London, London, UK

\subsection{6/gutjpl-2019-BSGAbstracts.129}

Introduction Patients with depression are at higher risk of later being diagnosed with Inflammatory Bowel Disease (IBD). Some speculate depression results in a pro-inflammatory state, predisposing to the development of IBD. An alternative explanation may be that patients experience undiagnosed gastrointestinal (GI) symptoms for long periods of time, during which they become depressed before receiving a delayed diagnosis of IBD. We aimed to determine if rates of depression are higher in individuals who subsequently develop IBD compared to the background population after adjustment for GI symptoms.

Methods Using a nationally representative research database, we identified incident cases of IBD diagnosed from 19982016. A non-IBD control group was matched $4: 1$ for age and sex. Our primary outcome measures were the prevalence of depression, antidepressant medication (ADM) use and GI symptoms in the year of IBD diagnosis and each of the ten years before. Controls were assigned the IBD diagnosis date of their matched IBD patient as a pseudo-diagnosis date. We used multivariable logistic regression to determine the risk of having depression and/or using an $\mathrm{ADM}$ in each of the ten years before IBD diagnosis/pseudo-diagnosis among IBD patients compared with controls. To prevent confounding by delayed of IBD we adjusted for the presence of GI symptoms in that year. Other covariates included age, sex, socioeconomic status and smoking status.

Results We identified 19555 cases of IBD and 78114 non-IBD controls. Depression was more prevalent among IBD patients compared with controls even 9 years prior to diagnosis $(2.1 \%$ vs $1.8 \%, \mathrm{p}=0.03)$. There was an excess of ADM use among IBD patients compared with controls as long as 9 years before diagnosis $(4.9 \%$ vs $4.4 \%, \mathrm{p}=0.03)$. GI symptoms were more common among IBD patients even 10 years before diagnosis $(6.3 \%$ vs $4.4 \%, p<0.01)$. Multivariable regression demonstrated patients who went on to be diagnosed with IBD were more likely to use ADMs as far as 7 years before diagnosis (OR 1.12, 95\%CI 1.02-1.22, $\mathrm{p}=0.01$ ).

Conclusions This is the first study to demonstrate the higher prevalence of depression and ADM use in IBD patients as long as 9 years before diagnosis. Some patients have GI symptoms up to a decade before receiving a diagnosis of IBD, suggesting delayed diagnosis is longer and more common than previously believed. Even after adjusting for the presence of GI symptoms, the higher risk of ADM use significantly predates IBD diagnosis, suggesting depression may play a part in mediating the onset of IBD.

\section{ATH-08 THE LARGEST SINGLE-CENTRE EXPERIENCE WITH STENT THERAPY IN CROHN'S DISEASE - A REPORT ON OUTCOMES}

\footnotetext{
${ }^{1,2}$ Ronit Das *, 'Rajeev Singh, 'Said Din, ${ }^{1,2}$ Jonathan Lund, ${ }^{1}$ Rajesh Krishnamoorthy, ${ }^{1}$ Stephen Hearing, ${ }^{1}$ Bernard Norton, ${ }^{1}$ Jessica Williams, ${ }^{1}$ Deepika Chilkunda, ${ }^{1}$ Catherine Fraser, ${ }^{1,2,3}$ Andrew Goddard, ${ }^{1}$ Andrew Cole. ${ }^{1}$ Royal Derby Hospital, Derby, UK; ${ }^{2}$ University of Nottingham, Nottingham, UK; ${ }^{3}$ Royal College of Physicians, London, UK
}

\subsection{6/gutjnl-2019-BSGAbstracts.130}

Introduction Intestinal strictures in Crohn's disease (CD) produce significant morbidity and are the commonest indication for surgery. Removable stent therapy is emerging as an effective, low complication, stricture resolution technique. (Das et al. 2018, Attar et al 2017) We present the clinical outcomes of the largest, single centre series, of CD patients undergoing removable 'self-expanding-metal-stent'(SEMS) therapy for stricture resolution.

Methods Symptomatic CD patients were assessed with MR enterography. Short $(\leq 6 \mathrm{~cm})$, fibrotic, ileocolonic and anastomotic strictures were approved for stent therapy from an IBD MDT setting. Strictures were assessed, and only endoscopically impassable strictures treated. Inaccessible or inflammatory strictures were excluded. The Hannaro Diagmed 'HRC-20-080230', partially covered SEMS was deployed under endoscopic and fluoroscopic guidance. Stent retrieval occurred at 6-10 days post insertion. Clinical IBD data was collected. Post-

Abstract ATH-07 Figure 1 Prevalence of depression before IBD diagnosis compared with controls 